\title{
A Case of Septic Portal Vein Thrombosis (Pylephlebitis)
}

\author{
Erin Carnish, MD, Andrew Garrett, MD, Mary Slome, MS I
}

\section{INTRODUCTION}

Pylephlebitis, defined as suppurative thrombosis of the portal vein often associated with bacteremia, is a rare and serious complication of intra-abdominal infections. Prior to antibiotics, the mortality rate of this disease approached $100 \% .1$ The veins adjacent to the infection are the first to be involved, with later spread to the portal vein and possibly the mesenteric and splenic veins. ${ }^{2-3}$ Extension into the mesenteric veins can lead to bowel necrosis and increased morbidity and mortality. ${ }^{2-4}$

\section{CASE PRESENTATION}

A 63-year old male with a history of hypertension presented to the emergency department with one week of progressively worsening malaise, chills, severe fatigue, and right upper quadrant abdominal pain. He was tachycardic with a heart rate of 120 and hypotensive to 70/30 mm Hg. Exam revealed a mildly distended abdomen with tenderness to palpation in the right upper quadrant and right lower quadrant. Laboratory studies were significant for a leukocytosis with bandemia and an elevation of the total bilirubin and alkaline phosphatase. Abdominal CT scan showed mucosal thickening involving the rectosigmoid colon. Nodular rounded lesions containing bubbles of air, concerning for multiple abscesses, ran the length of the recto-sigmoid colon and extended into the mesentery. $\mathrm{A}$ thrombus was noted in the main portal vein and superior mesenteric vein. There was heterogeneity in the left hepatic lobe consistent with multiple hepatic abscesses. Of note, the patient had a recent colonoscopy showing multiple sigmoid diverticulae. He was started on broadspectrum antibiotics with vancomycin and piperacillintazobactam. He briefly required vasopressor support. A heparin drip was also started to manage the portal vein and superior mesenteric vein thrombosis. Blood cultures eventually grew group $C$ streptococcus, and vancomycin was discontinued per the susceptibility pattern. The patient was treated in the hospital for 14 days and was discharged on oral amoxicillin-clavulanic acid for 14 additional days. During his stay, the patient was bridged to warfarin with a goal INR of 2-3.

\section{DIFFERENTIAL DIAGNOSIS}

Upon presentation, the patient met severe sepsis criteria and the focus was on resuscitation. The initial radiology report was misinterpreted as concerning for sigmoid cancer with hepatic metastasis, though the portal vein thrombosis was correctly identified upon re-examination with the staff radiologist.

\section{OUTCOME AND FOLLOW-UP}

Despite the initial delay in diagnosis, the patient was still treated appropriately given his presentation. Surgery was consulted; however, since there were multiple small abscesses and no single large area to drain, it was decided to repeat imaging after two weeks of antibiotic treatment. On the repeat $C T$, the abscesses had resolved, but the portal vein thrombosis persisted. The patient showed great improvement over his hospital course, and was doing well at his one month follow-up with his primary care doctor. He was treated with warfarin for a six month course.

\section{DISCUSSION}

The most common presentation of pylephlebitis is fever and abdominal pain. ${ }^{4}$ Laboratory abnormalities generally include leukocytosis with a left shift and elevations in alkaline phosphatase ${ }^{5,6}$ Our patient fit these criteria.

Infections such as appendicitis and diverticulitis are the most frequent predisposing conditions to pylephlebitis, though other intra-abdominal infections have also been implicated ${ }^{1.7}$

Blood cultures are positive in $23-88 \%$ of patients. ${ }^{1-4}$ When positive, organisms are usually bowel flora with 
Bacteroides fragilis and Escherichia coli being the most common. ${ }^{2-4}$ This patient grew group C streptococcus, which is somewhat less common, and his source was diverticulitis. The diagnosis depends on identifying an abdominal source of infection with an accompanying portal vein thrombus ${ }^{8-11}$ CT is most useful in both respects. ${ }^{5,89}$ Much of the increase in incidence of this disease can likely be attributed to the more prevalent use of CT. ${ }^{8-12}$

Once a diagnosis is made, the patient should be started on parenteral antibiotics, usually a third generation cephalosporin, a fluoroquinoline plus metronidazole, an extended spectrum penicillin or carbapenem for four to six weeks. ${ }^{1,13}$ Once clinical improvement is observed, the patient can be transitioned to oral antibiotics. Usually, the recommended oral regimen is metronidazole plus a flurorquinolone; ${ }^{13}$ however, under the guidance of an infectious disease specialist and the culture sensitivities, this patient was discharged on amoxicillin-clavulanic acid. Sometimes the infectious source may need drainage, but otherwise surgical intervention is not indicated for this condition. ${ }^{14}$ There is no substantial evidence based medicine on the use of anticoagulation. 1,3,13 $^{1,1}$

Limited data shows that anticoagulation may have a role in the case of mesenteric vein thrombosis to prevent bowel ischemia. ${ }^{15}$ Since this patient had SMV thrombosis, the decision was made to anticoagulate. While there is little data to support the use of anticoagulation, these are situations in which it could potentially decrease morbitity and mortality on a case-by case basis.

\section{KEY POINTS}

The mortality of pylephlebitis is estimated to be $11-32 \%$. 1,3,5 Prompt diagnosis and treatment are necessary for a good outcome. This diagnosis should be considered in all patients with abdominal pain and fever who are found on CT to have an intra-abdominal infection with a portal vein thrombus. Draw blood cultures and treat with antibiotics for 4-6 weeks. ${ }^{9}$

\section{REFERENCES}

1. Plemmons RM, Dooley DP, Longfield RN. Septic thrombophlebitis of the portal vein (pylephlebitis): diagnosis and management in the modern era. Clin Infect Dis 1995; 21: 1114.

2. Kasper DL, Sahani D, Misdraji J. Case records of the Massachusetts General Hospital. Case 25-2005. A 40-year-old man with prolonged fever and weight loss. N Engl J Med 2005; 353: 713.

3. Kanellopoulou T, Alexopoulou A, Theodossiades G, et al. Pylephlebitis: an overview of noncirrhotic cases and factors related to outcome. Scand J Infect Dis 2010; 42: 804

4. Baril N, Wren S, Radin R, et al. The role of anticoagulation in pylephlebitis. Am J Surg 1996; 172: 449.

5. Saxena R, Adolph M, Ziegler JR, et al. Pylephlebitis: a case report and review of outcome in the antibiotic era. Am J Gastroenterol 1996; 91: 1251

6. Joly $V$, Belmatoug N, Sibert A, et al. Septic thrombophlebitis of the portal vein. Clin Infect Dis 1996; 23: 417.12

7. Tung JY, Johnson JL, Liacouras CA. Portal-mesenteric pylephlebitis with hepatic abscesses in a patient with Crohn's disease treated successfully with anticoagulation and antibiotics. J Pediatr Gastroenterol Nutr 1996; 23: 474.

8. Harch JM, Radin RD, Yellin AE, Donovan AJ: Pylethrombosis. Serendipitous radiologic diagnosis. Arch Surg 1987; 122:1116-1119.

9. Balthazar EJ, Gollapudi P: Septic thrombophlebitis of the mesenteric and portal veins: CT imaging. J Comput Assist Tomogr 2000; 24: 755-760

10. Dean JW, Trerotola SO, Harris VJ, et al. Percutaneous management of suppurative pylephlebitis. J Vasc Interv Radiol 1995; 6: 585.

11. Van Gansbeke D, Avni EF, Delcour C, Engelholm L, Struyven J. Sonographic features of portal vein thrombosis. AJR Am J Roentgenol 1985; 144: 749-52.

12. Gajendran M, Muniraj T, Yassin M. Diverticulitis complicated by pylephlebitis: a case report. J Med Case Rep. 2011 Oct 10; 5: 514.

13. Duffy FJ Jr, Millan MT, Schoetz DJ Jr, Larsen CR. Suppurative pylephlebitis and pylethrombosis: the role of anticoagulation. Am Surg 1995; 61: 1041

14. Nouira K, Bedioui $H$, Azaiez $O$, Belhiba H, Messaoud MB, Ksantini R, Jouini M, Menif E. Percutaneous drainage of suppurative pylephlebitis complicating acute pancreatitis. Cardiovasc Intervent Radiol. 2007 Nov-Dec; 30(6): 1242-4

15. Chang TN, Tang L, Keller K, et al. Pylephlebitis, portal-mesenteric thrombosis, and multiple liver abscesses owing to perforated appendicitis. J Pediatr Surg 2001; 36: $19-21$ 